\title{
DNA Hybridization Catalysts and Catalyst Circuits
}

\author{
Georg Seelig ${ }^{1}$, Bernard Yurke $^{1,2}$, and Erik Winfree ${ }^{1}$ \\ ${ }^{1}$ California Institute of Technology, Pasadena, CA 91125, USA \\ 2 Bell Laboratories, Murray Hill NJ 07974, USA \\ \{seelig, yurke, winfree\}@dna.caltech.edu
}

\begin{abstract}
Practically all of life's molecular processes, from chemical synthesis to replication, involve enzymes that carry out their functions through the catalysis of metastable fuels into waste products. Catalytic control of reaction rates will prove to be as useful and ubiquitous in DNA nanotechnology as it is in biology. Here we present experimental results on the control of the decay rates of a metastable DNA "fuel". We show that the fuel complex can be induced to decay with a rate about 1600 times faster than it would decay spontaneously. The original DNA hybridization catalyst [15] achieved a maximal speed-up of roughly 30. The fuel complex discussed here can therefore serve as the basic ingredient for an improved DNA hybridization catalyst. As an example application for DNA hybridization catalysts, we propose a method for implementing arbitrary digital logic circuits.
\end{abstract}

\section{Introduction}

DNA has proven to be a highly versatile material for building artificial nanoscale devices. Among the devices already realized experimentally are DNA motors $[6,19,7]$, DNA walkers [12,13], DNA fuels [15], DNA catalysts [15] and selfassembled two dimensional crystals [16]. It is interesting to ask to what degree DNA alone can reproduce the richness of molecular biology and whether an alternative "DNA-only" world is conceivable. In fact, proposals that the history of life must include a time when nearly all the functions of life were subserved by RNA - the RNA World - have been given serious attention and are now widely accepted [3].

Biological systems exhibit complex and programmed behaviors. These behaviors are encoded by sets of specifically interacting molecules, such as DNA and proteins. The interactions among the molecules in such biochemical networks are akin to wires in electronic circuits. If we set our eyes on creating an artificial "cell" containing only DNA-based structures we need to design similar DNA-based biochemical networks that allow the components of the artificial cellular machinery to interact and communicate.

Biochemical circuits in biological systems almost universally rely on enzyme activity to carry out their function. It thus seems natural to use DNA catalytic systems as basic building blocks for a synthetic DNA-based circuit. While ribozymes are the best known example of nucleic acids with catalytic activity (such as DNA-cleaving or RNA-cleaving DNA enzymes $[11,1,14]$ ), we here want 
to use an alternative and entirely different type of DNA based catalytic system, namely a DNA hybridization catalyst [15]. DNA hybridization catalysts are rationally designed molecules that catalyze the conformational rearrangement of other DNA complexes. The catalytic system typically consists of two components: (i) a metastable "fuel", i.e. a DNA complex forced into a state from which it can not decay spontaneously into its true minimal energy configuration ("waste product"), and (ii) the actual catalyst which makes a fast pathway available for the transformation of the fuel complex into waste. The term fuel is used here, since the free energy that becomes available when the metastable complex decays can in principle be used to perform mechanical work. The fuel complex could for example serve as an energy source for a autonomous DNA-based molecular motor.

The first example of a DNA hybridization catalytic system was devised by Turberfield et al. [15]. There, the metastable fuel consisted of a pair of complementary strands of DNA that were inhibited from hybridization by inducing at least one of the strands to acquire a loop-like structure through partial hybridization with another strand of DNA. The inhibition was thought to be due to the difficulty a complementary strand of DNA has in threading its way through the loop to form duplex DNA. Catalytic speed-up was achieved by a short strand of DNA that binds on one side of the loop via a toehold and opens the loop through three-strand branch migration.

In Sec. 2 we describe this system in detail and propose modifications which promise to significantly enhance catalytic activity and applicability. In Sec. 3 we propose a scheme for linking such DNA hybridization catalysts into networks capable of performing complex logic. Finally, in Sec. 4 we will present experimental results on the construction and characterization of a DNA fuel complex and the catalytic control over the decay of the fuel complex.

\section{Improving the DNA hybridization catalyst}

Catalytic control of chemical reactions is essential for the design of autonomous behavior in biochemical systems. Two features are necessary for high performance of a catalytic system: programmability of the interactions so that catalyst systems can be tailored to a given task, and high ratios between the rates of catalyzed and uncatalyzed reactions. We expect that the former criterion will pose little difficulty for DNA hybridization catalysts, since catalyst design should be relatively insensitive to the specific choice of nucleotide sequence, once essential constraints are incorporated into the design process. The more interesting problem is to design a system with a high catalysis ratio.

Previous work has identified systematic approaches to the control of DNA hybridization kinetics. A key phenomenon, studied in [10,4,17, 18], is strand displacement by branch migration (Fig. 1a). Here, the reaction $P \bar{S}+S \rightarrow S \bar{S}+P$ is thermodynamically favorable, because of the additional base pairs that are formed. The kinetics of the reaction depends upon the length of the singlestranded overhang, known as a toehold. The toehold is where $S$ initially binds to $P \bar{S}$, and the longer it is, the more likely that the reaction will enter a branch migration phase prior to dissociation. Branch migration consists of isoenergetic 
steps where the final base pair of $P$ to $\bar{S}$ is replaced by a base pairing of $S$ to $\bar{S}$, thus moving the branch point by a random walk process $[9,8]$. When the branch point reaches the left side of the complex, strand $P$ dissociates. This is an essentially irreversible step, because there is no toehold for $P$; although, due to spurious "toeholds" produced by DNA breathing, the reaction can occur at low rates. Using fluorescence of a fluorophore/quencher pair to read out the bulk fraction of molecules in which the fluorophore and quencher were near each other (as in the random-coil state of $Q$ ), Yurke et al. [18] measured an exponential acceleration of reaction kinetics due to toehold lengths from 0 (where the rate constant is $\sim 1 / \mathrm{M} / \mathrm{s}$ ) to 6 (where the rate approaches that of ordinary hybridization, namely $\sim 10^{6} / \mathrm{M} / \mathrm{s}$ ). The principle of strand displacement mediated by toeholds will be essential also for the more complicated systems considered below.
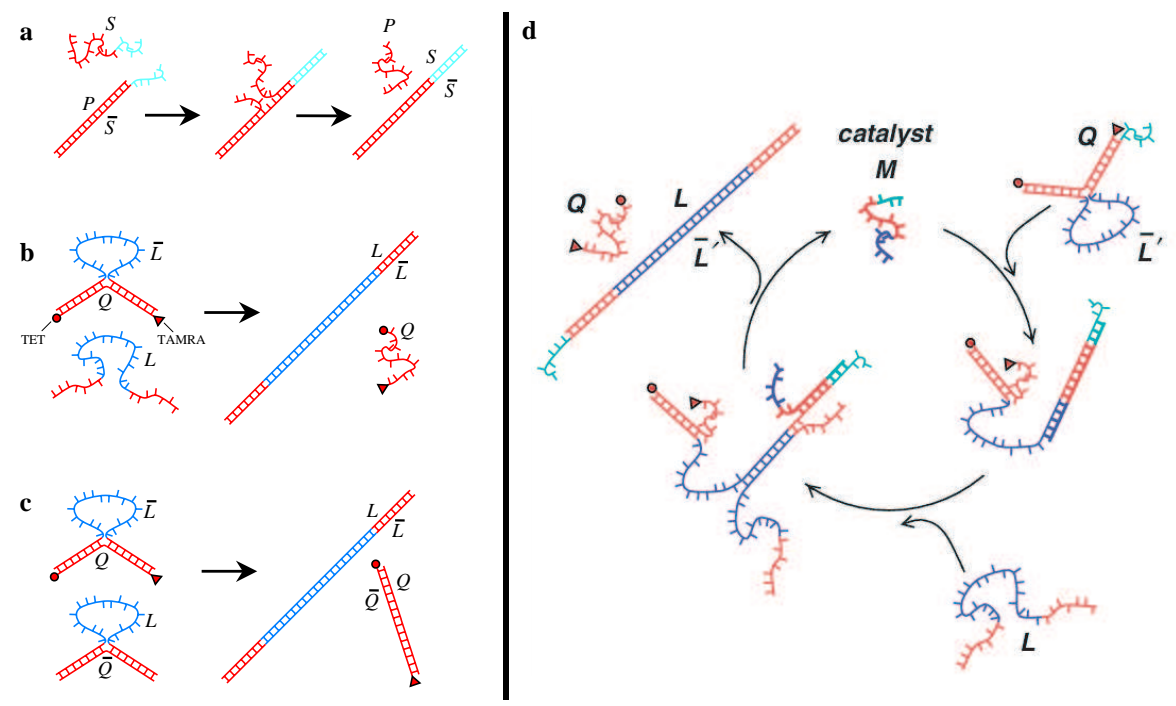

Fig. 1. (a) Single-stranded toehold allows rapid displacement of $P$ by $S$. (b) The $Q \bar{L}+L \rightarrow Q+L \bar{L}$ reaction is slow. (c) The $Q \bar{L}+L \bar{Q} \rightarrow Q \bar{Q}+L \bar{L}$ reaction is even slower. (d) Strand $M$ catalyzes the $Q \bar{L}+L \rightarrow Q+L \bar{L}$ reaction.

We now turn to DNA hybridization catalysts [15], where the reaction rate is controlled not by a permanent structural change in the molecules (such as adding a toehold or changing the length of a toehold) but rather by an additional strand, namely the catalyst strand. A precondition for such a system is a metastable DNA complex or pair of complexes for which a thermodynamically favorable but kinetically inaccessible configuration exists. A catalyst for this reaction must make available a fast pathway to the thermodynamically favorable state. Thus, the metastable complexes represent an energy source that can in principle be coupled to other reactions, with timing controlled by the catalyst. 
An example is shown in Fig. 1b. As described in Ref. [15], the 40 nucleotide loop region was presumed to be tightly coiled, preventing hybridization in that domain. The second-order rate constant for this reaction was measured to be $\sim 420 / \mathrm{M} / \mathrm{s}$, which is $\sim 10^{4}$ times slower than the rate for the hybridization of unconstrained single-stranded DNA, such as $L$ and $\bar{L}$. A catalyst for this reaction (Fig. 1d) was proposed and demonstrated in Ref. [15], where a 30-fold speed-up due to the catalyst strand $M$ was measured.

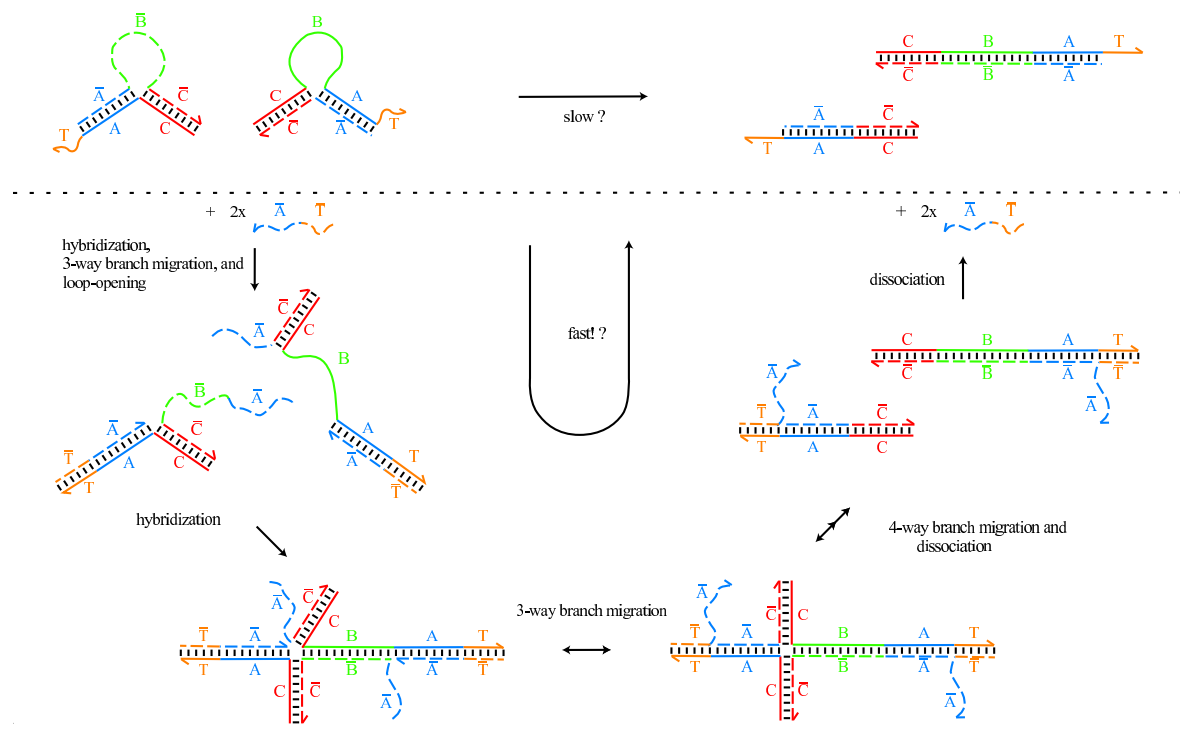

Fig. 2. Two copies of strand $M$ catalyze the $Q \bar{L}+L \bar{Q} \rightarrow Q \bar{Q}+L \bar{L}$ reaction of Fig. 1c. Here, subsequences of each strand are explicitly labeled, and the necessary toeholds are added. Thus, $M=\bar{T} \bar{A}, Q=C A T, \bar{L}=\bar{A} \bar{B} \bar{C}, \bar{Q}=\bar{A} \bar{C}$, and $L=C B A T$. The dotted line separates uncatalyzed reactions from those that occur only in the presence of catalyst strand $\bar{T} \bar{A}$.

To increase the catalysis ratio, however, we need a pair of metastable fuel complexes that have an even lower spontaneous hybridization rate. An obvious candidate is the system consisting of the molecules $Q \bar{L}$ and $L \bar{Q}$ shown in Fig. 1c where now both long strands $L$ and $\bar{L}$ are forced into a loop. A preliminary study reported in Ref. [15] established an upper limit of $3 / \mathrm{M} / \mathrm{s}$ for the second order rate constant of this reaction. The similarity with the singly protected system (Fig. 1b) suggests that catalysis of the hybridization reaction $Q \bar{L}+L \bar{Q} \rightarrow$ $Q \bar{Q}+L \bar{L}$ will be possible.

Before turning to the experimental implementation (see Sec. 4) we will discuss one potential pathway for a catalyzed reaction between two loops (see Fig. 2) whose logic is based on the conclusions of [15]: The presumed compact state of the loop prevents interaction with single strands or other loops. To enable the loop-loop hybridization reaction, both of the fuel complexes must be opened, 
exposing both loop regions. This is accomplished by one molecule of the catalyst strand binding to each fuel complex. Thus, in the second hybridization step, both loop regions are open and available for hybridization. Once the combined complex has been formed, 3-way branch migration brings the conformation to the point where 4-way branch migration of the $C$ arms may take place. After this final step, the complex dissociates into two inert waste products, from which the catalyst strands can dissociate due to the weak binding in the short toehold domain.

Unlike the original catalyst, which made use of 3-way branch migration only, the improved catalyst makes use of both 3-way branch migration (where individual steps are typically $\sim 10 \mu \mathrm{s}$ ) as well as 4 -way branch migration (in which individual steps are typically $\sim 100 \mathrm{~ms}$ depending upon reaction conditions) [9, 8]. Short 4-way branch migration reactions are typically completed in a few seconds, so we don't expect this to be the rate-limiting step. However, in another study we have discovered that initiation of branch migration at a junction can be surprisingly slow [20]. The results of that study suggest that a three nucleotide toehold would significantly enhance the reaction rates. To achieve this, we truncate the terminal three nucleotides of the catalyst $\bar{T} \bar{A}$, leaving 3 nt of $A$ unpaired to serve as a toehold to initiate 3 -way branch migration.

Note that the reaction pathway shown in Fig. 2 is by no means the only one possible. In fact, the analysis of complex DNA hybridization pathways such as this one poses profound challenges, because the number of possible intermediate complex conformations (as measured by secondary structure) can be exceedingly large. For example, branch migration reactions in different parts of the molecule can occur at different rates and complete in different orders. Furthermore, what are shown as unstructured single-stranded regions may fold into weakly-structured states that can significantly affect the rates of reactions. Also, entirely unexpected reactions can in principle occur. One approach to these issues is to use stochastic models of secondary structure kinetics [2] to identify unexpected pathways or steps at which the reaction stalls. Initial simulations have demonstrated that the pathway shown here can in fact go to completion with sequences based on the original catalyst system.

\section{$3 \quad$ Logic gates and circuits}

In biological organisms, systems of catalysts regulate fundamental biological pathways. The ability of catalysts to regulate the activity of other catalysts allows complex logic to be implemented. The potential programmability of DNA hybridization catalysts may be ideal for the construction of logic circuits. However, for this to be accomplished, the catalysis of one pair of fuel complexes must be somehow coupled to the catalysis of a second pair of fuel complexes which have unrelated sequences. Our proposal for accomplishing this builds on the improved catalyst design by modifying one of the fuel complexes to contain an additional strand, $\bar{X}$, hybridized to the inside of the loop, as shown in Fig. 3. This strand is then released by strand displacement during an intermediate 3way branch migration step in the reaction. Since the sequence of strand $\bar{X}$ is unrelated to the other parts $(A, B$, and $C)$ of the catalyst design, we are free 
to choose its sequence to be the catalyst for an otherwise unrelated downstream hybridization reaction. By making the two toehold sequences distinct, a different catalyst strand is required to open each of the fuel complexes, and only presence of both of the input strands allows the reaction to go to completion. This effects AND-gate logic, i.e., the output $\bar{X}$ is produced if and only if both $\bar{T} \bar{A}$ and $\bar{S} \bar{A}$ are present, which we write as $\operatorname{Gate}(\bar{T} \bar{A} \& \bar{S} \bar{A} \Rightarrow \bar{X})$.

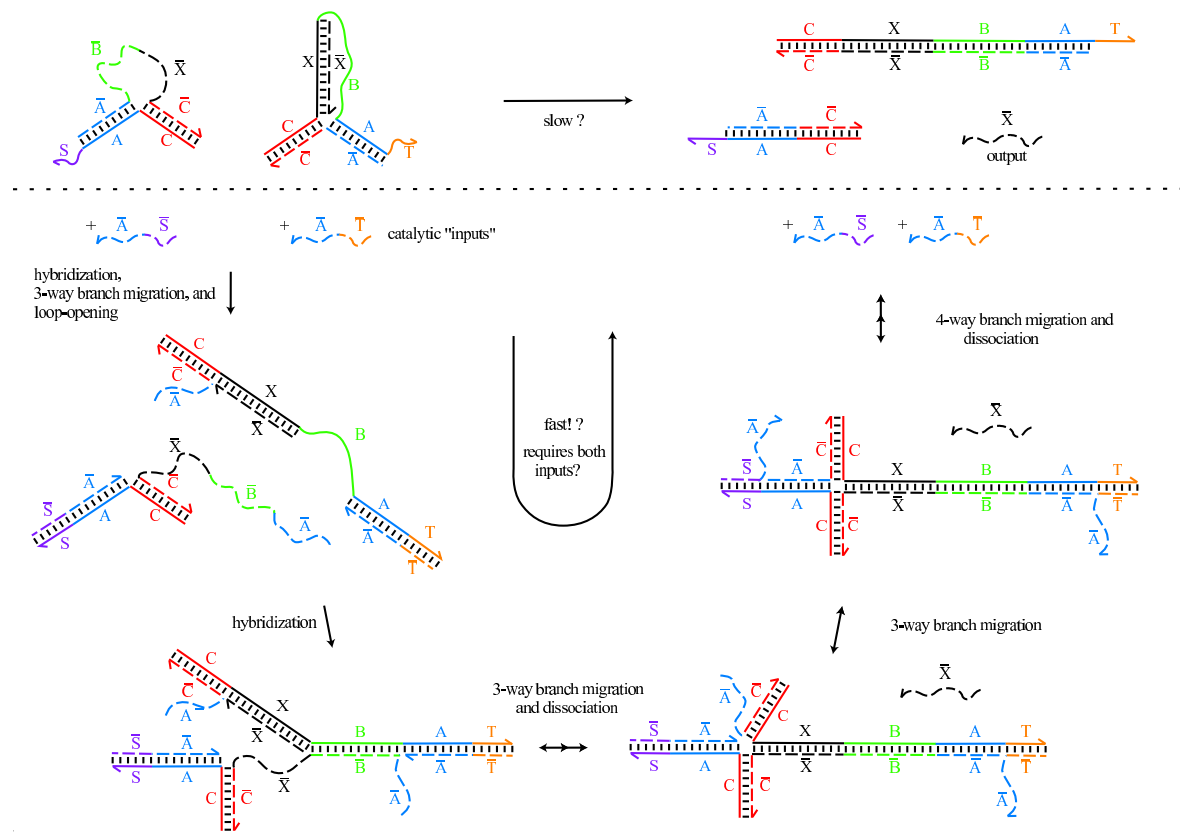

Fig. 3. An AND-gate catalyst constructed via two modifications of the improved catalyst shown in Fig. 2: (1) two distinct toehold sequences $S$ and $T$ guard access to the two loop-opening reactions required in the catalysis pathway; and (2) one of the loops contains a hybridized "output" strand, $\bar{X}$, which is released by strand displacement during the formation of $L \bar{L}$. Thus, the overall reaction can be written as $\operatorname{Gate}(\bar{T} \bar{A} \& \bar{S} \bar{A} \Rightarrow \bar{X})$, indicating that $\bar{X}$ is produced as a single-stranded species only if both $\bar{T} \bar{A}$ and $\bar{S} \bar{A}$ are present. The dotted line separates uncatalyzed reactions from those that occur only in the presence of input strands.

A simple variation of this gate results in OR-gate logic: give the toeholds from the $A$ stems of both loops the same sequence $T$, and create analogous toeholds on both $C$ stems with sequence $S$. Now, either input $\bar{T} \bar{A}$ or input $\bar{S} \bar{C}$ will be sufficient to trigger the catalytic step and release the output strand $\bar{X}$. We write this as $\operatorname{Gate}(\bar{T} \bar{A} \mid \bar{S} \bar{C} \Rightarrow \bar{X})$.

To implement any desired computation, a universal gate such as NAND is needed. AND and OR provide a universal basis only for monotone circuits, which can directly implement a strict subclass of all boolean functions, specifically those for which an input bit flipping from 0 to 1 can never cause the output to flip 
from 1 to 0 . However, this seeming limitation is lifted if we allow a "dual rail" input representation, wherein distinct signals $x_{i}^{+}$and $x_{i}^{-}$are used to represent " $x_{i}=0$ " and " $x_{i}=1$ ". Either $x_{i}^{+}=1$ or $x_{i}^{-}=1$, but not both; however, both $x_{i}^{+}$and $x_{i}^{-}$can be 0 , indicating that bit $x_{i}$ has not yet been computed. To see that an arbitrary function $x_{n}=f\left(x_{1}, x_{2}, \ldots x_{m}\right)$ can be implemented with monotone gates, consider a circuit of NAND gates that implements $f(\cdot)$. Replace each gate $x_{k}=x_{i}$ NAND $x_{j}$ by the pair of monotone gates $x_{k}^{+}=x_{i}^{-}$OR $x_{j}^{-}$ and $x_{k}^{-}=x_{i}^{+}$AND $x_{j}^{+}$. Computation in the new circuit begins with all variable at zero, then the appropriate input variables are flipped to 1 and subsequent downstream gates are evaluated. (Note: if a gate is evaluated prematurely, and both inputs to an OR or AND gate are 0, nothing happens to the output either.) As soon as the signal reaches the output gate $n$, either $x_{n}^{+}$or $x_{n}^{-}$flips to 1 , giving the result of the computation.

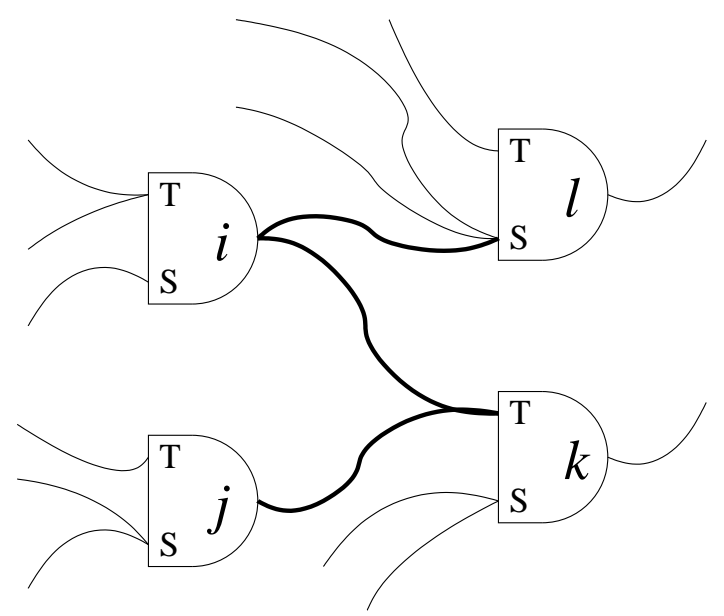

Fig. 4. A monotone logic circuit with AND gates and wired OR. The two inputs to each AND gate are distinguished. In this diagram, if either gate $i$ or gate $j$ outputs 1 , the "T" input of gate $k$ is activated. To translate this circuit to a DNA hybridization catalyst network, we require one AND-gate catalyst for each wire in the diagram. For example, the three thick wires become the three catalyst gates $\operatorname{Gate}\left(\bar{T}_{i} \bar{A}_{i} \& \bar{S}_{i} \bar{A}_{i} \Rightarrow \bar{S}_{l} \bar{A}_{l}\right)$, $\operatorname{Gate}\left(\bar{T}_{i} \bar{A}_{i} \& \bar{S}_{i} \bar{A}_{i} \Rightarrow \bar{T}_{k} \bar{A}_{k}\right)$, Gate $\left(\bar{T}_{j} \bar{A}_{j} \& \bar{S}_{j} \bar{A}_{j} \Rightarrow \bar{T}_{k} \bar{A}_{k}\right)$.

It is straightforward to use DNA hybridization AND-gates and OR-gates to implement arbitrary digital circuits using this "dual-rail" monotone logic. Indeed, because we have proposed no mechanism for getting rid of a catalyst once it is released, the monotone logic property is essential for the circuits we could build this way. To further minimize the size of constructed circuits, and to show that the shared sequence requirement of the AND-gate catalyst's inputs causes no difficulties, we use two optimizations. First, all OR gates may be eliminated and replaced by a "wired-OR"; i.e., each gate that outputs one of the OR gate's inputs can now instead directly produce the OR gate's output 
itself. The resulting circuit can be pictured as in Fig. 4. The inputs to the new AND gate $i$ are distinguished as type " $T$ " or type " $S$ ", corresponding to whether the DNA input strands will be $\bar{T}_{i} \bar{A}_{i}$ or $\bar{S}_{i} \bar{A}_{i}$ respectively, where the subscript $i$ indicated that distinct DNA sequences will be used for each gate. Now, for each distinct output wire from each AND gate, we construct an AND-gate catalyst with the given inputs and the given output for that wire. Noting that arbitrary fan-out can be achieved, this completes the construction.

In a large circuit consisting of DNA hybridization catalyst gates, input is provided by adding the catalysts corresponding to 1 or 0 input variables, as appropriate. This will trigger the release of the logically correct intermediate catalysts for intermediate gates, and finally, the release of either the 1 output strand or the 0 output strand. Recognizing that the uncatalyzed rate of each hybridization reaction is non-zero, we must consider that eventually every intermediate and every output catalyst species will be released. Thus, to evaluate the probable reliability of circuits constructed this way, understanding the timing of reactions is essential: will the 1 output strand or the 0 output strand be produced

first? It is critical, for example, that the presence of a single catalyst input to an AND gate will not significantly accelerate the reaction. In some cases a biochemical AND gate, with both inputs driven by the same species, can function as a restoring element to correct small errors [5]; it remains to be seen to what extent the sigmoidal input/output curve expected for the AND-gate catalyst can be used to reduce errors in this context.

It is remarkable that active biochemical logic can in principle be accomplished by DNA without the assistance of any enzymes. Note however that we have only shown how to construct feed forward circuits; it is an open question whether the general scheme presented here can be adapted to the case of feedback circuits, where signals can be turned off and on dynamically.

\section{Experimental implementation}

From the discussion in the previous section it is clear that the experimental realization of a DNA-based catalyst with a large catalysis ratio is a necessary precondition for the implementation of a DNA-logic circuit. Our preliminary experimental results indicate that the system outlined in Sec. 2 may indeed work as a catalyst with a remarkably large catalysis ratio. However, the experiment also shows several interesting deviations from the theoretical scheme.

Typical experimental data for the reaction between the two loops $Q \bar{L}$ and $L \bar{Q}$ (in the following we will use the notation introduced in Fig. 1) is shown in Fig. 5. In this experiment, the strands $\bar{L}$ and $Q$ are unlabeled, strand $\bar{Q}$ is fluorescence-labeled with the fluorophore TAMRA at the $3^{\prime}$-end and $L$ is labeled with an Iowa Black quencher at the $5^{\prime}$-end. Thus, the reactant $L \bar{Q}$ is dark, while the product $Q \bar{Q}$ is fluorescent. The loops are formed in a slow anneal. The experiment shown was performed at a constant temperature $T=20^{\circ} \mathrm{C}$ and with a concentration $c=0.5 \mu \mathrm{M}$ for the two loops. The reaction is initiated when the $Q \bar{L}$-solution is added to the $L \bar{Q}$-solution. An increase in fluorescence intensity is a direct measure of the rate of dissolution of the $L \bar{Q}$-loop as the reaction $Q \bar{L}+L \bar{Q} \rightarrow Q \bar{Q}+L \bar{L}$ proceeds. In the example shown in Fig. 5 we 


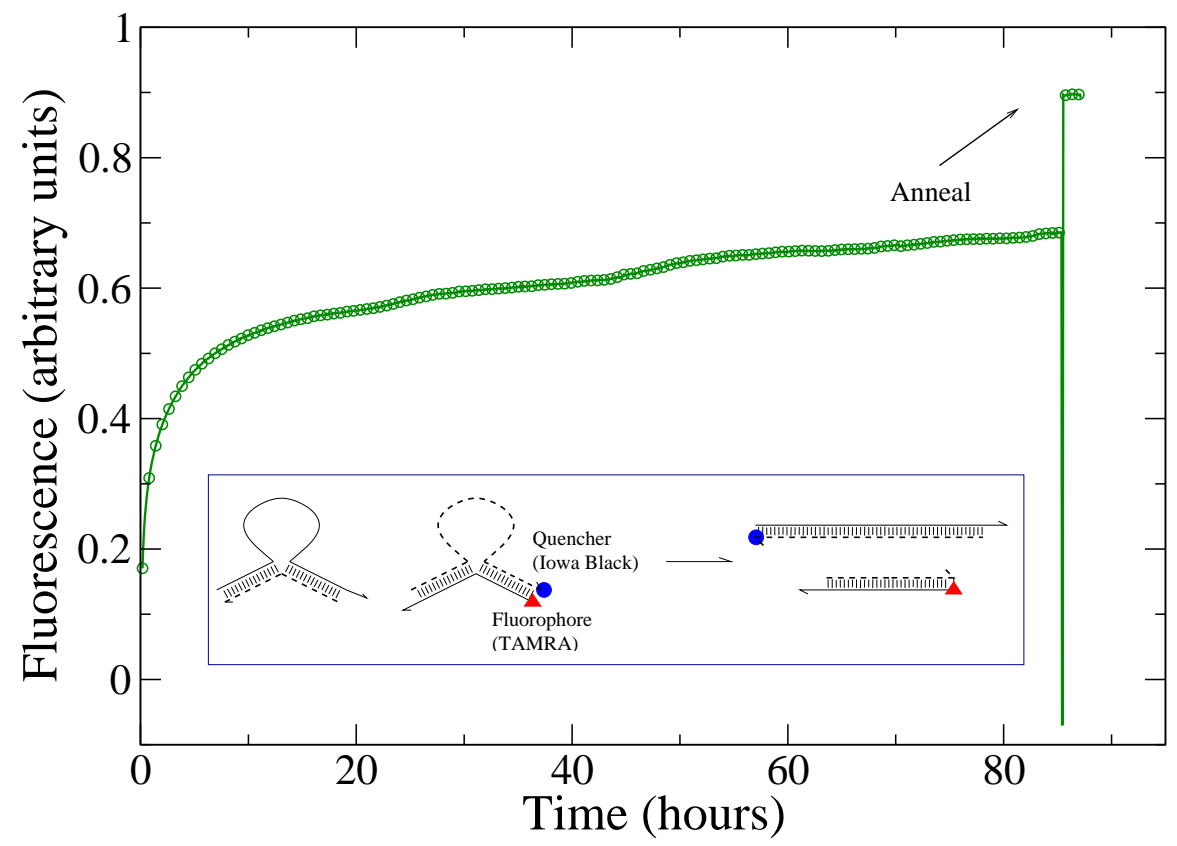

Fig. 5. The figure shows the long-time behavior of a stoichiometric mixture of the two loops $Q \bar{L}$ and $L \bar{Q}$. Strands $\bar{L}$ and $Q$ are unlabeled, strand $\bar{Q}$ is fluorescencelabeled with the fluorophore TAMRA at the $3^{\prime}$-end and $L$ is labeled with an Iowa Black quencher at the $5^{\prime}$-end (see inset). In the initial state fluorescence is quenched. The increase in fluorescence intensity is a measure of the progress of the reaction $Q \bar{L}+L \bar{Q} \rightarrow Q \bar{Q}+L \bar{L}$. Remarkably, only the anneal performed after $\sim 86$ hours brings the reaction to completion. This indicates the presence of a metastable compound in the solution. The experiment is performed in SPSC buffer $(\mathrm{pH} 6.5,1 \mathrm{M} \mathrm{NaCl})$ at a temperature $T=20^{\circ} \mathrm{C}$.

followed the reaction over more than three days and, as can be seen from the figure, the fluorescence intensity changes very little after the first few hours or so. One might thus conclude that after this point almost all the available loops have reacted and only inert segments of double-stranded DNA are present in the solution. However, surprisingly, annealing the sample up to $80^{\circ} \mathrm{C}$ for 5 minutes at the end of the measurement and subsequently remeasuring the fluorescence intensity shows an increase in fluorescence of around 25 percent.

From this experiment we can draw two main conclusions: First, as the relatively rapid initial increase in fluorescence indicates, the mixture of the two loops $Q \bar{L}$ and $L \bar{Q}$ is less stable than one might have expected. In fact, a more detailed analysis shows that the reaction $Q \bar{L}+L \bar{Q} \rightarrow Q \bar{Q}+L \bar{L}$ initially progresses at a rate comparable to that of the reaction $\bar{L} Q+L \rightarrow Q+L \bar{L}$ where only one of the two long strands is protected (see Fig. 1b). Taken alone, this would indicate that the catalyst proposed in Sec. 2 is at best a minor improvement over the simpler system of Ref. [15]. Our second finding, however, is more intriguing: Even after several days the reaction has not gone to completion, as indicated by the large 
jump in fluorescence intensity upon annealing. What is more, an electrophoresis gel shows that directly before the anneal three species are present in the solution mixture (see Fig. 6). Two of them are the expected reaction end products $L \bar{L}$ and $Q \bar{Q}$ while the third one is a complex with a mobility roughly half that of a single loop. After the anneal, only $L \bar{L}$ and $Q \bar{Q}$ are found.

(a)

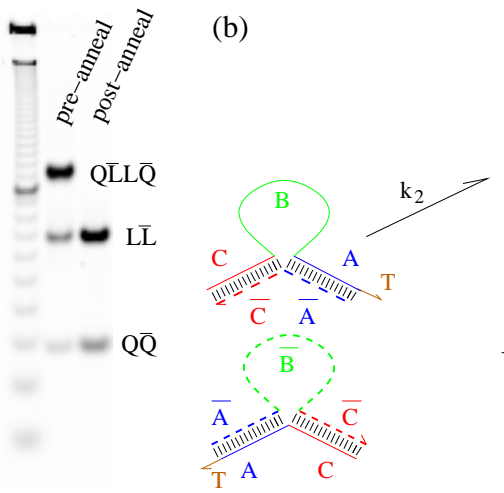

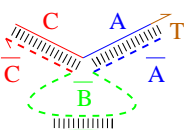

iाIIIIII"

B

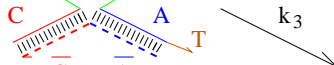

$=\overline{\mathrm{C}} \quad \overline{\mathrm{A}}$

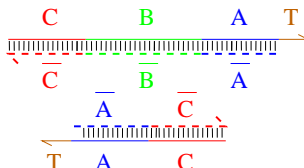

Fig. 6. (a) Products of the reaction between the two loops $Q \bar{L}$ and $L \bar{Q}$. The reaction was left to proceed for about twelve hours $\left(\mathrm{T}=20^{\circ} \mathrm{C}\right.$, TAE buffer, $\left.\mathrm{pH} 8,12.5 \mathrm{mM} \mathrm{Mg}^{++}\right)$ before the gel was run. Left lane: ladder with ten base pair spacing. Middle lane: the three bands correspond to the metastable fuel complex $Q \bar{L} L \bar{Q}$ (lowest mobility) and to the double stranded end products $L \bar{L}$ and $Q \bar{Q}$ (highest mobility). Right lane: After an anneal, only the stable end products $L \bar{L}$ and $Q \bar{Q}$ are found. No bands corresponding to unreacted loops are seen (the mobility of the unreacted loops is comparable to that of a $55 \mathrm{mer}$ ). (b) Proposed reaction pathway: The two loops $Q \bar{L}$ and $L \bar{Q}$ can either decay directly into the end products $L \bar{L}$ and $Q \bar{Q}$ (rate constant $k_{1}$ ) or via a metastable complex. In our model, $k_{2}$ is the second order rate constant for the formation of the intermediate complex and $k_{3}$ is the first order rate constant describing its subsequent decay. Subsequences of each strand are explicitly labeled: $Q=C A T, \bar{L}=\bar{A} \bar{B} \bar{C}$, $\bar{Q}=\bar{A} \bar{C}$, and $L=C B A T$.

While it is difficult to determine the exact structure of the low-mobility species, we have verified that all four strands $(L, \bar{L}, Q$ and $\bar{Q})$ used for the formation of the loops are also present in this complex. It is therefore plausible that the complex is formed due to strong interactions between the single stranded regions of the two loops.

Two competing reactions appear to be taking place when solutions of the two loops are mixed. In one of them, the final double-stranded minimum-energy compounds are formed directly. Let the rate constant for this reaction be $k_{1}$. The second, parallel reaction proceeds via a metastable intermediate which only slowly decays into the end products $L \bar{L}$ and $Q \bar{Q}$. We model the rate constants for these two steps as $k_{2}$ and $k_{3}$, respectively (see Fig. 6 ). The putative reactions 


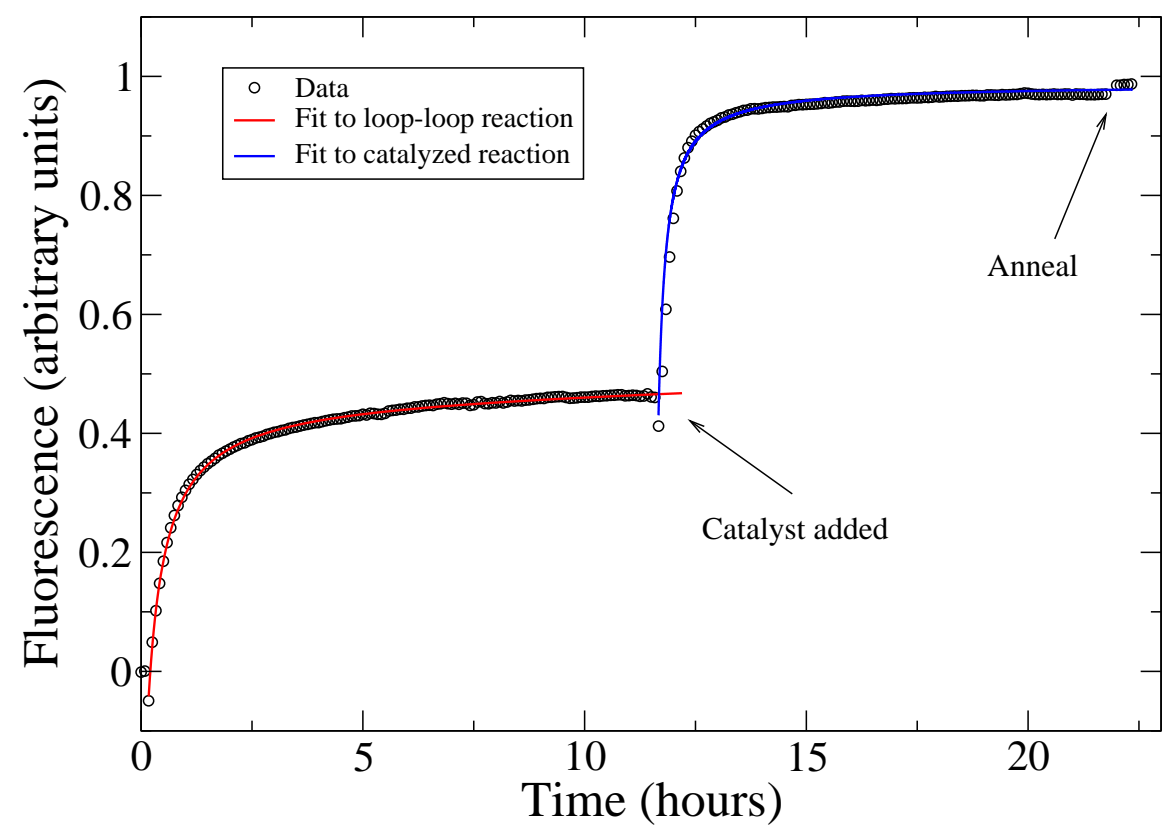

Fig. 7. Catalysis of the reaction between the two loops $Q \bar{L}$ and $L \bar{Q}$. The experiment was performed in TAE buffer $\left(\mathrm{pH} 8,12.5 \mathrm{mM} \mathrm{Mg}^{++}\right)$at a temperature $T=20^{\circ} \mathrm{C}$ with an initial concentration $c=0.5 \mu \mathrm{M}$ for both loops. The locations of the dye label and quencher are as indicated in the inset of Fig. 5. Catalyst strand $M$ (concentration $c=0.3 \mu \mathrm{M})$ is added after approximately 12 hours. After the addition of the catalyst the reaction goes to completion and a final anneal increases the fluorescence only marginally. The full lines are fits to the data using the model described in the text. For the rate constants we use the numerical values $k_{1}=690 / \mathrm{M} / \mathrm{s}, k_{2}=670 / \mathrm{M} / \mathrm{s}$, $k_{3}=10^{-6} / \mathrm{s}$ and $k_{4}=5300 / \mathrm{M} / \mathrm{s}$.

taking place can be summarized as follows:

$$
\begin{aligned}
& Q \bar{L}+L \bar{Q} \stackrel{k_{1}}{\rightarrow} Q \bar{Q}+L \bar{L}, \\
& Q \bar{L}+L \bar{Q} \stackrel{k_{2}}{\rightarrow} Q \bar{L} L \bar{Q} \stackrel{k_{3}}{\rightarrow} Q \bar{Q}+L \bar{L} .
\end{aligned}
$$

To estimate the rate constants $k_{1}, k_{2}$ and $k_{3}$ we use a simple mathematical model for the reaction kinetics. The reaction given in Eq. 1 and the first step of the reaction in Eq. 2 are assumed to be second order, while the second step of the reaction in Eq. 2 is treated as a first order reaction. The fluorescence concentration is assumed to be proportional to the concentration of $Q \bar{Q}$ and it is further assumed that the fluorescence remains quenched in the complex $Q \bar{L} L \bar{Q}$. From the slow decay of the fluorescence intensity at long times it is clear that the rate for the decay of $Q \bar{L} L \bar{Q}$ must be small.

The metastable complex $Q \bar{L} L \bar{Q}$ is dissolved very easily upon addition of the catalyst strand $M$ as is shown in Fig. 7. The loops used in this reaction are dye-labeled as described above and mixed stoichiometrically at a concentration $c=0.5 \mu \mathrm{M}$. Catalyst strand $M$ at a concentration of $c=0.3 \mu \mathrm{M}$ was added 


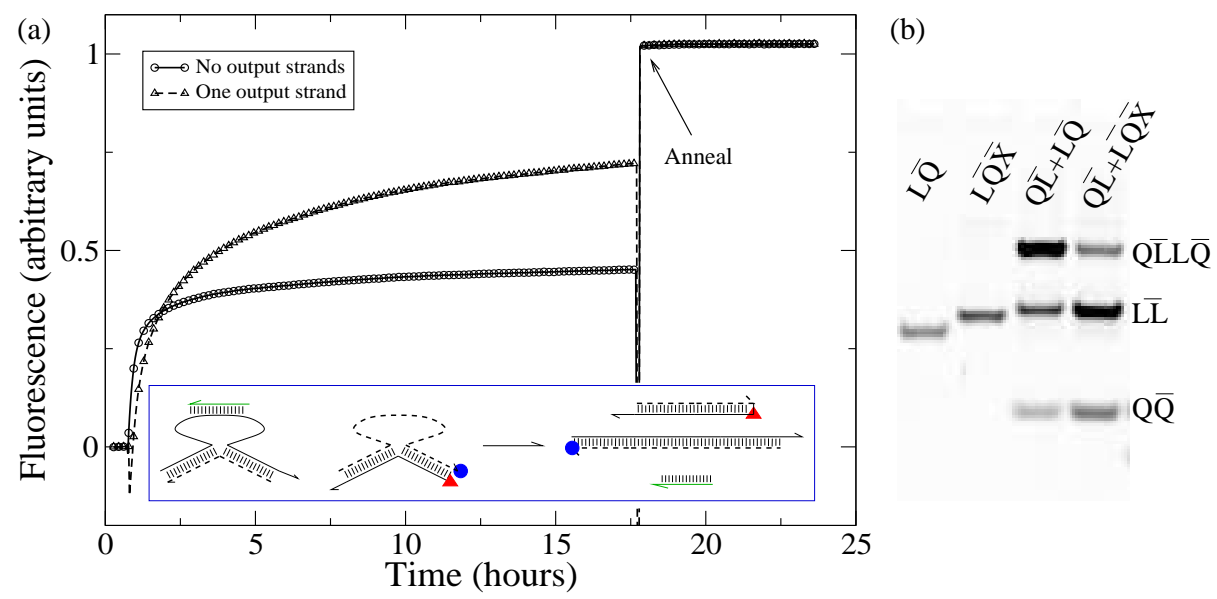

Fig. 8. (a) Comparison of the reactions between the two unmodified loops $Q \bar{L}$ and $L \bar{Q}$ and the reaction between $Q \bar{L}$ and $L \bar{Q} \bar{X}$. The experiment was performed in TAE buffer $\left(\mathrm{pH} 8,12.5 \mathrm{mM} \mathrm{Mg}^{++}\right.$) at a temperature $T=20^{\circ} \mathrm{C}$ with an initial reactant concentration $c=0.5 \mu \mathrm{M}$. The locations of the dye label, quencher and output strand $\bar{X}$ are as indicated in the inset. (b) Reactants and reaction products for the reaction between $Q \bar{L}$ and $L \bar{Q} \bar{X}$. Far left lane: Loop $Q \bar{L}$. Center left lane: Modified loop $L \bar{Q} \bar{X}$. Center right lane: Products of the reaction between $Q \bar{L}$ and $L \bar{Q}$. Far right lane: Products of the reaction between $Q \bar{L}$ and $L \bar{Q} \bar{X}$.

after about 12 hours. The reaction then very rapidly went to completion. In fact, a final anneal did not markedly change the fluorescence intensity in contrast to our previous result.

To estimate the reaction speed up we need to compare the rate constants before and after catalyst addition. The uncatalyzed reaction is modeled as outlined above (see Fig. 6 and Eqs. 1 and 2): The data in Fig. 7 is well approximated by our model with $k_{1}=690 / \mathrm{M} / \mathrm{s}, k_{2}=670 / \mathrm{M} / \mathrm{s}$ and $k_{3}=10^{-6} / \mathrm{s}$ (see Fig. 7). The values for the rate constants given here should be considered as order of magnitude estimates. As we have already mentioned previously, the rate constants $k_{1}$ and $k_{2}$ are of the same order of magnitude as the rate constant for the reaction $L \bar{Q}+\bar{L} \rightarrow \bar{Q}+L \bar{L}$ (see Ref. [15]). The reaction after addition of catalyst strand can be fitted with a simple second order rate law with a rate constant $k_{4}=5.3 \times 10^{3} / \mathrm{M} / \mathrm{s}$ (see Fig. 7). Since the catalyst concentration is $c=0.3 \mu \mathrm{M}$ the half-time $\tilde{t}_{1 / 2}$ for this reaction is $1 / k_{4} c=625 \mathrm{~s}$. The half time $t_{1 / 2}$ for the uncatalyzed decay of the metastable compound, on the other hand, is $t_{1 / 2}=1 / k_{3}=10^{6} \mathrm{~s}$. If we define the reaction speed up as the ratio of the half times for the decay of the metastable compound before and after addition of catalyst strand we obtain $t_{1 / 2} / \tilde{t}_{1 / 2}=1600$.

In Sec. 3 we proposed a modification of the original dual catalyst design where an additional strand $\bar{X}$ is hybridized to the inside of the loop. This strand is released when the loops react and can itself act as a catalyst in a downstream reaction. The proposal for implementing logic gates outlined in Sec. 3 relied on two assumptions: (i) that the two loops remain stable and independent in solu- 
tion and (ii) that there is a clear separation of timescales between the reactions taking place in the absence and presence of input strands. The surprising existence of the intermediate $Q \bar{L} L \bar{Q}$ foreshadows that the scheme discussed in Sec. 3 will have to be modified at least in part.

Intuitively, one would expect strand $\bar{X}$ to hinder loop-loop interactions and thus to suppress complex formation. That this is indeed the case can be seen from the preliminary experimental data shown in Fig. 8. There, the reaction between the modified loop $L \bar{Q} \bar{X}$ and loop $Q \bar{L}$ is compared to the reaction between $L \bar{Q}$ and $Q \bar{L}$. In our experiment, the output strand $\bar{X}$ has a length of 21 bases (the same length as the catalyst strand) and its sequence is complementary to the central part of $L$. To form the modified loop $L \bar{Q} \bar{X}$ the output strand is added to a solution of preformed loops $L \bar{Q}$. The left two lanes in the gel of Fig. $8 \mathrm{~b}$ show the band corresponding to the unmodified loop $Q \bar{L}$ (far left) and to the (less mobile) modified loop $L \bar{Q} \bar{X}$ (center left).

The fluorescence data (Fig. 8a) shows that the reaction with one modified loop proceeds slower than that between the unmodified loops but also goes closer to completion. An explanation for the latter is provided by the gel in Fig. 8b, center right and far right lanes, which shows that in the presence of output strands relatively more loops participate in a reaction that leads to end product formation than to complex formation. Contrary to our assumptions, even in the modified reaction essentially all the loops seem either to decay into doublestranded end products or to form metastable complexes and do not remain in solution in their original form. While no band corresponding to free output strand $\bar{X}$ is seen in the gel, it seems plausible that $\bar{X}$ is released in both the decay and the complex formation reaction. (This is confirmed indirectly since the bands corresponding to all reaction products migrate at the same speed in both cases.)

\section{Discussion}

The slow long-term increase in fluorescence intensity we observed for $Q \bar{L}+L \bar{Q}$ is in qualitative agreement with the preliminary results on loop-loop reactions reported in [15]. A quantitative comparison is difficult since reactant concentration, location of dye and quencher within the molecules, as well as the dye-quencher pair used, differ between our work and the work reported in [15]. However, our results shed light on the actual origin of the observed stability. The discovery that $Q \bar{L}$ and $L \bar{Q}$ form a stable complex also leads to the view that the reaction depicted in Fig. 1b proceeds through the formation of a bound complex between $Q \bar{L}$ and $L$ that then slowly decays to $L \bar{L}$ and $Q$. This differs from the intuitive view, expressed earlier, that the reaction is inhibited because of tight coiling of the loop. Furthermore, it now seems probable that the catalysis cycle shown in Fig. 1d proceeds by a different pathway (for substoichiometric catalyst strand concentrations): $Q \bar{L}$ first forms a complex with $L$, then the catalyst strand accelerates its decay into $Q$ and $L \bar{L}$.

Further work is needed to demonstrate catalytic speed-up of the reaction leading to the decay of the metastable compound. So far, we have only measured the reaction speed-up in the case where catalyst strand and complex are 
mixed roughly stoichiometrically. The result thus obtained can be considered an estimate for an upper bound on the reaction rates. However, to show that the catalyst strand indeed acts catalytically, it will be necessary to demonstrate turnover - i.e. to show that the reaction goes to completion for sub-stoichiometric amounts of catalyst strand and that in this regime the reaction rate increases (linearly) with catalyst concentration.

To implement an OR gate or an AND gate one would have to demonstrate that adding input strands to a mixture of $L \bar{Q} \bar{X}$ and $Q \bar{L}$ speeds up the release of the output strand $\bar{X}$. However, contrary to the situation encountered in the catalysis experiments discussed above, it is fruitless to add an input strand to the solution after the metastable complex has formed, because presumably all output strands have already been released at this point. Alternatively, catalyst strands could be added when the two loops are mixed initially, and their effectiveness measured by a speed-up in the release of the output strands. For such an experiment it seems most convenient to choose an intramolecular dye/quencher configuration that allows one to directly monitor the release of the output strand. It seems probable that at least the relatively simpler OR gate can be implemented according to the scheme outlined here, as all it requires is the speed-up of the initial reaction between $L \bar{Q} \bar{X}$ and $Q \bar{L}$ in the presence of either of two input strands. The on/off ratio for such a gate can be expected to be comparable to the ratio between the catalyzed and uncatalyzed reactions of Ref. [15] which was found to be about 20 to 30 . However, for implementing more complicated circuits in which several gates are linked, it seems more promising to completely redesign the constituent gates using the metastable complex explicitly as a starting point. In this way, one could hope to design gates with an on/off ratio of the order of one thousand.

Acknowledgments. Thanks to Ben Rahn, Jeremy Leibs, Joseph Schaeffer, Jongmin Kim, Dave Zhang, and especially Paul Rothemund for stimulating discussion and help preparing figures and simulations. GS was supported by the Swiss National Science Foundation, EW was supported by NSF CAREER Grant No. 0093486, NSF ITR Grant No. 0113443, and GenTel.

\section{References}

1. N. Carmi, S. R. Balkhi, and R. R. Breaker. Cleaving DNA with DNA. Proceedings of the National Academy of Sciences, 95:2233-2237, 1998.

2. C. Flamm, W. Fontana, I. Hofacker, and P. Schuster. RNA folding at elementary step resolution. RNA, 6:325-338, 2000.

3. R. F. Gesteland, T. R. Cech, and J. F. Atkins. The RNA world. Cold Spring Harbor Laboratory Press, Cold Spring Harbor, New York, 1999.

4. C. Green and C. Tibbetts. Reassociation rate-limited displacement of DNA strands by branch migration. Nucleic Acids Research, 9:1905-1918, 1981.

5. M. O. Magnasco. Chemical kinetics is Turing universal. Physical Review Letters, 78(6):1190-1193, 1997.

6. C. D. Mao, W. Q. Sun, Z. Y. Shen, and N. C. Seeman. A nanomechanical device based on the B-Z transition of DNA. Nature, 397:144-146, Jan. 14, 1999.

7. C. M. Niemeyer and M. Adler. Nanomechanical devices based on DNA. Angewandte Chemie International Edition, 41(20):3779-3783, 2002. 
8. I. G. Panyutin, I. Biswas, and P. Hsieh. A pivotal role for the structure of the Holliday junction in DNA branch migration. The EMBO Journal, 14(8):18191826, 1995.

9. I. G. Panyutin and P. Hsieh. Kinetics of spontaneous DNA branch migration. Proceedings of the National Academy of Sciences, 91:2021-2025, 1994.

10. C. Radding, K. Beattie, W. Holloman, and R. Wiegand. Uptake of homologous single-stranded fragments by superhelical DNA. IV. branch migration. J. Mol. Biol., 166:825-839, 1977.

11. S. W. Santoro and G. F. Joyce. A general purpose DNA cleaving RNA enzyme. Proceedings of the National Academy of Sciences USA, 94:4262-4266, 1997.

12. W. B. Sherman and N. C. Seeman. A precisely controlled DNA biped walking device. Nano Letters, 4(7):1203-1207, 2004.

13. J. Shin and N. Pierce. A synthetic DNA walker for molecular transport. Journal of the American Chemical Society, 126(35):10834-10835, 2004.

14. M. N. Stojanovic, T. E. Mitchell, and D. Stefanovic. Deoxyribozyme-based logic gates. Journal of the American Chemical Society, 124:3555-3561, 2002.

15. A. J. Turberfield, J. C. Mitchell, B. Yurke, A. P. Mills, Jr., M. I. Blakey, and F. C. Simmel. DNA fuel for free-running nanomachines. Physical Review Letters, 90(11):118102-1-4, 2003.

16. E. Winfree, F. Liu, L. A. Wenzler, and N. C. Seeman. Design and self-assembly of two-dimensional DNA crystals. Nature, 394:539-544, 1998.

17. D. M. Wong, P. H. Weinstock, and J. G. Wetmur. Branch capture reactions: displacers derived from asymmetric PCR. Nucleic Acids Research, 19:2251-2259, 1991.

18. B. Yurke and A. P. Mills, Jr. Using DNA to power nanostructures. Genetic Programming and Evolvable Machines, 4:111-122, 2003.

19. B. Yurke, A. J. Turberfield, A. P. Mills, Jr., F. C. Simmel, and J. L. Neumann. A DNA-fuelled molecular machine made of DNA. Nature, 406:605-608, 2000.

20. D. Y. Zhang and J. Schaeffer. Personal communication, 2003. 